FACTA UNIVERSITATIS

Series: Mechanical Engineering Vol. 19, No 2, 2021, pp. 241 - 252

https://doi.org/10.22190/FUME201228011B

Original scientific paper

\title{
COMPUTATIONAL MICROSTRUCTURE-BASED ANALYSIS OF RESIDUAL STRESS EVOLUTION IN METAL-MATRIX COMPOSITE MATERIALS DURING THERMOMECHANICAL LOADING
}

\author{
Ruslan Balokhonov ${ }^{1}$, Varvara Romanova ${ }^{1}$, Eugen Schwab ${ }^{2}$, \\ Aleksandr Zemlianov ${ }^{1,3}$, Eugene Evtushenko ${ }^{1}$ \\ ${ }^{1}$ Institute of Strength Physics and Materials Science, SB, RAS, Russia \\ ${ }^{2}$ Doppelmayr Seilbahnen GmbH, Austria \\ ${ }^{3}$ National Research Tomsk State University, Russia
}

\begin{abstract}
A technique for computer simulation of three-dimensional structures of materials with reinforcing particles of complex irregular shapes observed in the experiments is proposed, which assumes scale invariance of the natural mechanical fragmentation. Two-phase structures of metal-matrix composites and coatings of different spatial scales are created, with the particles randomly distributed over the matrix and coating computational domains. Using the titanium carbide reinforcing particle embedded into the aluminum as an example, plastic strain localization and residual stress formation along the matrix-particle interface are numerically investigated during cooling followed by compression or tension of the composite. A detailed analysis is performed to evaluate the residual stress concentration in local regions of bulk tension formed under all-round and uniaxial compression of the composite due to the concave and convex interfacial asperities.
\end{abstract}

Key words: Structure of materials, Computer simulation, Metal-matrix composites, Residual stresses, Strain localization

\section{INTRODUCTION}

Wear is an extremely complex process during which material surface layers are subjected to a combination of different types of loading: tension, compression and shear. Non-homogeneous degradation of the material during wear is mainly caused by the geometrical stress concentration due to surface asperities [1-3] or microstructural

Received December 28, 2020 / Accepted February 08, 2021

Corresponding author: Ruslan Balokhonov

Institute of Strength Physics and Materials Science SB RAS 634055, Tomsk, pr. Akademicheskii, 2/4

E-mail: rusy@ispms.tsc.ru 
inhomogeneity [4], which is clearly expressed, for instance, in composite materials and coatings. The formation of composite coatings on metallic parts, from one hand, improves the strength and wear and corrosion resistance of their surface layers due to reinforcing particles. From the other hand, complex hierarchical structure of the composites, containing components of intricate irregular shapes with differing physical-mechanical properties: ceramic particles, metal matrices and substrates, transition zones between the matrix and particles, polycrystalline grains, etc., results in the stress concentration and plastic strain localization. Even minor changes in the structure of a composite can result in a change of the deformation and fracture mechanisms at different scale levels and appreciably affect its strength. The composite's structural inhomogeneity gives rise to generation of residual stresses (RSs) during manufacture and coating deposition [5-7], which influence the material strength and durability in its future service. Solutions of these problems can be directly associated with investigations of the stress concentration and strain localization mechanisms under thermal-mechanical loading of composites by the microstructurebased numerical simulation. An advantage of a numerical analysis over an experiment consists in a possibility of examining the stressed state evolution in the bulk of the composites and during their deformation.

The ceramic particles used in numerical simulations of metal-matrix composites and coatings, which explicitly include structure inhomogeneity into consideration, are mostly characterized either by an ideal round shape and the problem is solved in a two-dimensional formulation [8-11], or by a spherical shape [11-13] in a three-dimensional analysis. Such important issues as, for instance, the influence of the relative positions of ceramic particles, their dimensions and volume fractions on the composite's fracture character are reported in the current literature $[8,9]$. The discrete element method using a thermal expansion model taking into account damage effects and interfacial debonding was applied in [10] to simulate the thermal induced cracking due to thermal expansion mismatch between the constituent materials. The mechanisms of particle fracture are included into consideration in [11] as the processes of pore formation and coalescence. The influence of adhesion at the particle matrix interface is reported in $[12,13]$. In particular, tension of a composite with a single particle is simulated in [12], and tension-compression of a composite is numerically investigated in [13], taking into account the residual stresses developing in the composite after its quenching into water. A comparison of the stress and plastic strain distribution is performed including adhesion and without adhesion at the interfaces taken into consideration. An elastoplastic model based on a unit cell consisting of 8 subcells representing elastic particle and plastic matrix materials is developed in [14] to compute elastic moduli and residual stresses at both the constituent and composite levels. There are much fewer studies taking into account nonideal shapes of the reinforcing particles. 2D model metal-matrix composite with an interface zone between polyhedral ceramic particles and the metallic binder is studied in [15]. In [16], the effect of the composite's two-dimensional structure with polyhedral particles on the local characteristics of temperature distributions under thermal loading is discussed. Meaningful simulation of the composite's strain and damage in a three-dimensional structure with a few particles of regular polyhedral shapes is carried out in [17], without including any residual stresses, while in [18] effective elastic moduli and the coefficient of thermal expansion for the aluminum matrix reinforced by the particles of equal icosahedral shapes and sizes are predicted. Plastic strain localization and fracture across multiple spatial scales in metal-matrix composite and coated materials are investigated by solving two- [19-21] and three-dimensional [22] problems without taking into 
consideration cooling-induced RSs, while in [21] the 3D numerical analysis of the residual stress formation under cooling is performed.

The purpose of this work is to develop a technique for designing three-dimensional structures of metal-matrix composites and coatings including complex irregular shapes of reinforcing particles into consideration, which is observed in the experiments. Furthermore, using a computer simulation of cooling followed by compression or tension as an example, the aim is to investigate in detail the residual stress concentrations and their influence on the strength of the composites fabricated by ex-situ technologies, where the pre-synthesized particles are embedded into the matrix during a secondary process such as selective laser melting, stir casting, solid state sintering during hot pressing, etc.

\section{Microstructure Generation TeCHNIQUe FOR COMPOSITE AND COATED MATERIALS}

The initial step in the microstructure-base analysis is the development of procedures for an explicit inclusion of their three-dimensional structure into the model. In this study we present a straightforward method for constructing numerical models of metal-matrix composites. The reinforcing carbide particles have irregular angular shapes qualitatively similar to rock fragments (Fig. 1). Therefore, in developing geometrical models of composite materials and coatings having complicated microstructure it is proposed to use macroscopic naturally broken rock stones. This approach allows assuming a scale invariance of the natural mechanisms of spalling during mechanical grinding.

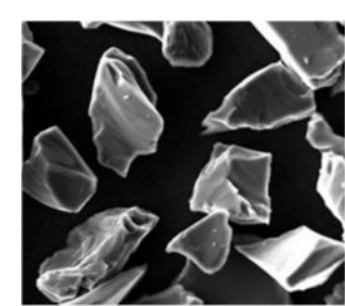

a)

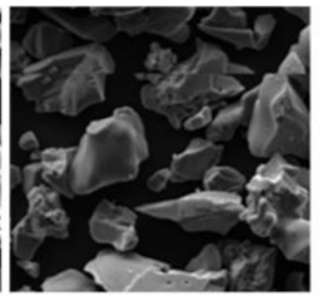

b)

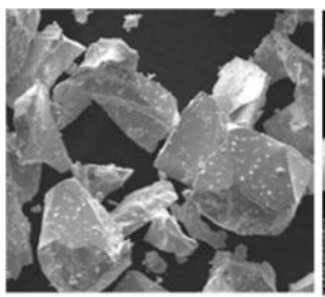

c)

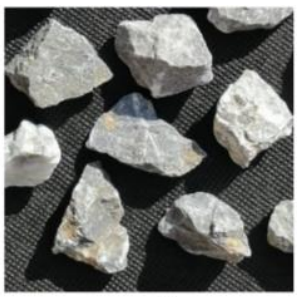

d)

Fig. 1 Examples of the boron (a), titanium (b) and tungsten carbide particles (c) and chip stones $(\mathrm{d})$

For this procedure, a few hundred chip stones were arbitrarily selected, which measured from 80 to $100 \mathrm{~mm}$ in their maximum section. In order to transform the stone surface into a 3D digital analog 3DSOM Pro software was used, which is readily available and sets minimum requirements to the hardware tools.

The following equipment was used in obtaining the initial data: illuminated rotary stage, photo-studio for filming the specimen surfaces, additional light sources, and camera on a tripod. All specimens were coated with thin layers of white paint in order to speed up the process of automated determination of their contours. After performing the required adjustment, about a hundred frames were obtained from every evenly rotating stone specimen in equal time intervals (Fig. 2a). 
a)

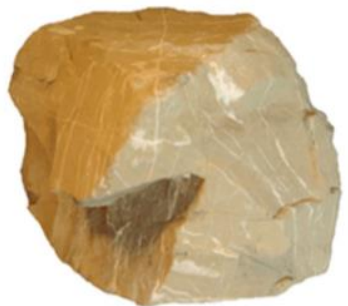

d)

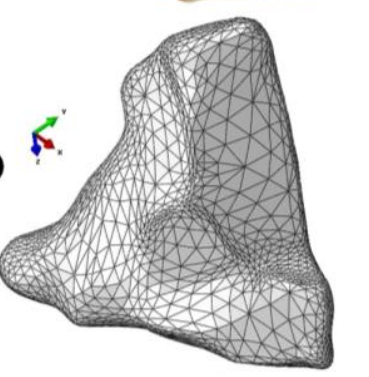

g)

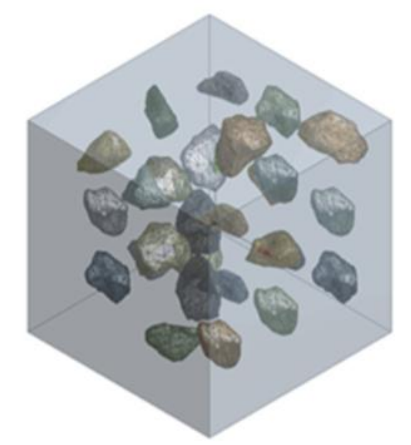

b)

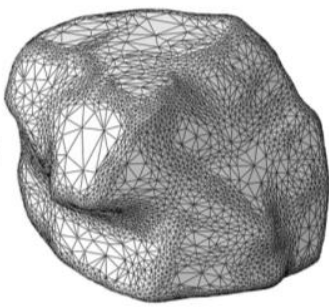

e)

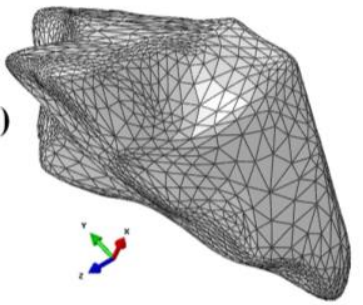

c)

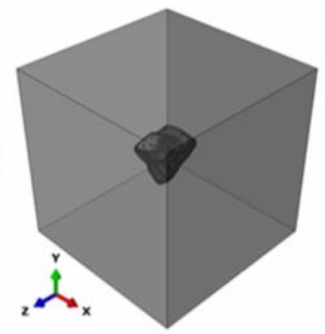

f)

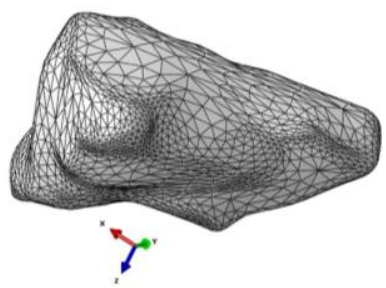

h)

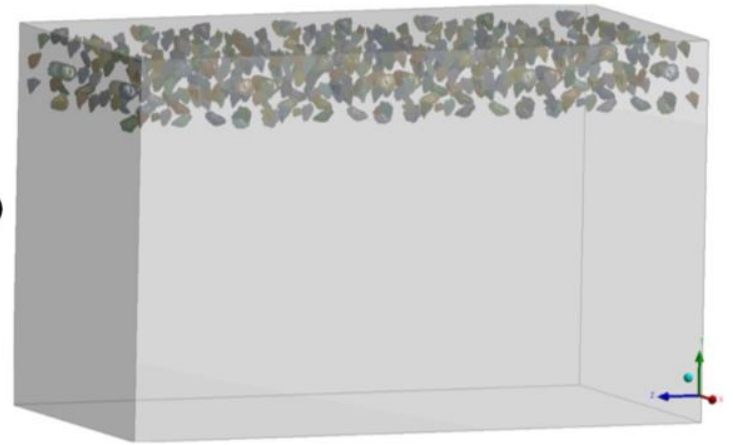

Fig. 2 Photo of a stone (a), numerical model of the stone-particle (b) and the particle embedded into a cubic aluminum microvolume (c), different side views of another particle (d-f) and models of the composite (g) and coated materials (h)

Then using 3DSOM Pro software 3-D models of these specimens were constructed. Their photographic images were imported as the initial data of a new project. The specimen surfaces were recognized in the manual and semiautomatic modes and separated from the image background and shadows, thus masks were created from the objects under study. Following this, the process of designing a three-dimensional model continued in an automatic mode via the recognition of the camera and specimen positions in space. For every specimen, a 3-D model was designed, wherein the number of surfaces was varied from 1000 to 10000 depending on the surface geometry complexity, so that no fewer than 50 facets corresponded to a structure irregularity. Examples of the stone models are presented in Figs. 2b, 2d-f. Using an STP file format, the resulting threedimensional surfaces of stones-particles were imported into ABAQUS and by a random spread of CSYS orientations and coordinates of the stone centers three-dimensional material structures with particles of irregular shapes of different scales (Figs. 2c, 2g and 2h) were designed. 


\section{SPECIAL ASPECTS OF RESIDUAL STRESS FoRMATION IN COMPOSITES DURING COOLING FOLLOWED BY MECHANICAL LOADING}

Consider the approach of multilevel numerical simulation with an explicit inclusion of the material structure by the example of evolution of residual stresses (RSs) generating in metal-matrix composites and coatings during their manufacture and subsequent use. A special attention would be given to the formation of local regions subjected to tensile stresses. It is in these regions that local defects and cracks can nucleate under highintensity thermo-mechanical loading, resulting in an early loss of macroscopic strength of the composites and parts manufactured from them. The formation of tensile regions under external compressive loading is a controversial issue requiring detailed studies. In this work in order to distinguish between the individual mechanisms of stress concentration under different types of thermo-mechanical loading three series of numerical experiments on deforming aluminum-titanium carbide composite were successively performed:

1. Uniaxial compression and tension of a specimen without its preliminary cooling (not including RSs into consideration). The results and conclusions made in this part are valid for the specimens subjected to, for instance, stress-relieving treatment.

2. Cooling from the melt to room temperature, which is often the case during manufacture of the composites under the conditions close to those of all-round compression.

3. Cooling followed by uniaxial compression or tension of the composite.

The focus was on the structure presented in Fig. 2c, with the volume fraction of the particle material being $1 \%$. It should be noted that in this case all the results obtained are valid for the composites and coatings with a small volume fraction of particles in the absence of their mutual influence. Three-dimensional static problems were solved numerically by the finite-element method using ABACUS. In the simulation of thermal loading, all faces of the representative volume element (RVE) presented in Fig. 2c are free from loading. We consider a steady-state process, wherein the temperature in all local regions of the RVE, including the matrix and particle materials, in every loading step is taken to be equal and is linearly decreased from the melting to room temperature from 660 to 24 degrees Celsius. Unlike a purely mechanical problem, during thermal loading the Duhamel - Neumann's relations are solved, for which an additional contribution into the cubic part of the stress tensor is included into consideration - in calculating the cubic strain a term responsible for thermal expansion is added

$$
\sigma_{i j}=\left(K-\frac{2}{3} \mu\right) \delta_{i j} \varepsilon_{k k}+2 \mu \varepsilon_{i j}-3 K \alpha\left(T-T_{0}\right) \delta_{i j}
$$

The results of simulations are aggregated in Fig. 3; they allow making the following conclusions. Firstly, it has been determined that local regions undergoing bulk tensile stress appear under compression of composites. These regions are formed as early as in the elastic stage of composite deformation in the areas of concavities of the interface between the less stiff matrix and stiffer particle, with the maximum pressure values observed in the concavities whose position is perpendicular to the direction of compression (Fig. 3a, C-type regions). These are the most vulnerable regions of possible nucleation of defects and cracks under high-intensity loading. Secondly, comparing the calculations for the elastic matrix-elastic particle and plastic matrix-elastic particle composites, a conclusion can be drawn that the volume fraction of tensile regions during uniaxial compression is larger in the case of plastic matrix than it is if the matrix is 
assumed to be elastic (Cf. Figs. 3a and 3b). Generalizing, this means that, other conditions being equal, single cracks are formed inside the particles in the ceramic composites, while in metal-matrix composites multiple cracking of the particles is possible.
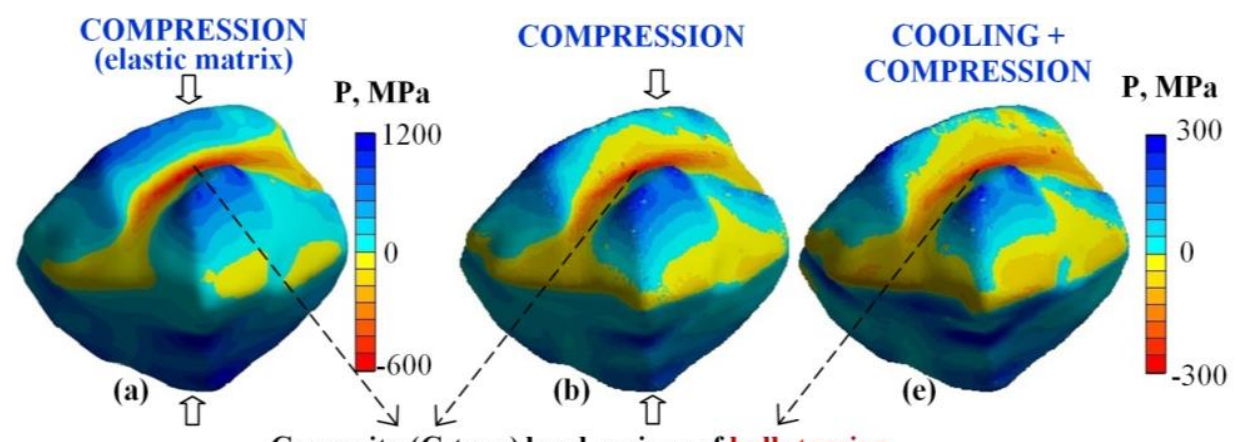

Concavity (C-type) local regions of bulk tension

Concavity (C-type) local regions of bulk compression
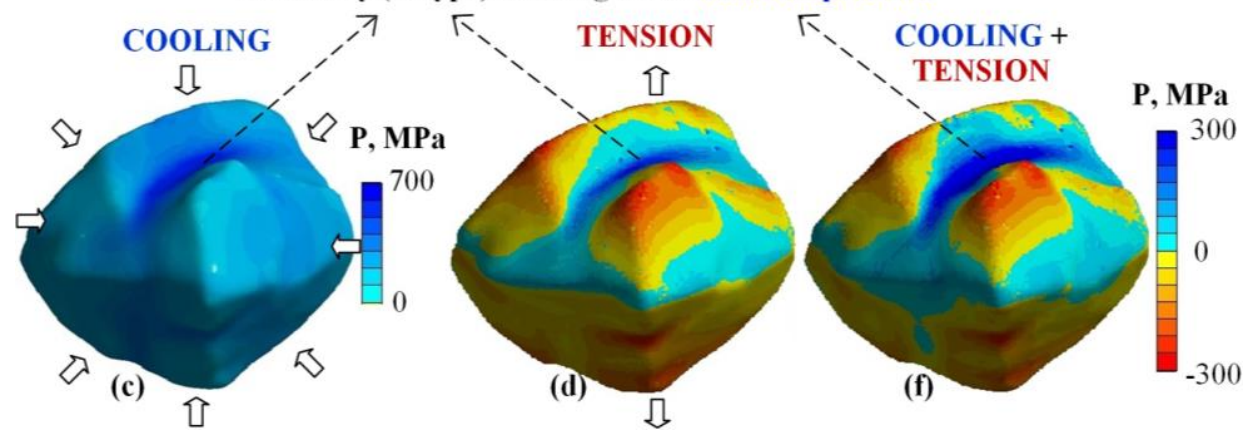

Fig. 3 Pressure in the elastic TiC particle under different loading of the Al-TiC composite shown in Fig. 2c: compression for the cases of elastic (a) and plastic matrices (b), cooling from $660^{\circ} \mathrm{C}$ to room temperature (c), tension (d) and cooling followed by compression (e) and tension of the composite (f)

A pressure distribution pattern in a ceramic particle during cooling is shown in Fig. 3c: the composite is compressed by about $1.6 \%$, i.e., to the same degree to which uniaxial compression was simulated (Fig. 3b). It has been determined here that unlike uniaxial compression, in which case different regions of the particle are subjected to both negative and positive pressures, during cooling of the structure no regions of bulk tension are formed in the particle at all, they are rather concentrated solely in the matrix (which will be shown in what follows). This conclusion drawn from the results of numerical simulations is entirely consistent with the experimental data [3]. Moreover, the average level of pressure is higher under all-round compression (cooling) than during uniaxial compression.

The respective pressure and equivalent plastic strain distributions in the aluminum matrix are presented in Fig. 4, showing only the matrix material without the particle. 
Computational Microstructure-Based Analysis of Residual Stress Evolution in Metal-Matrix Composite...247

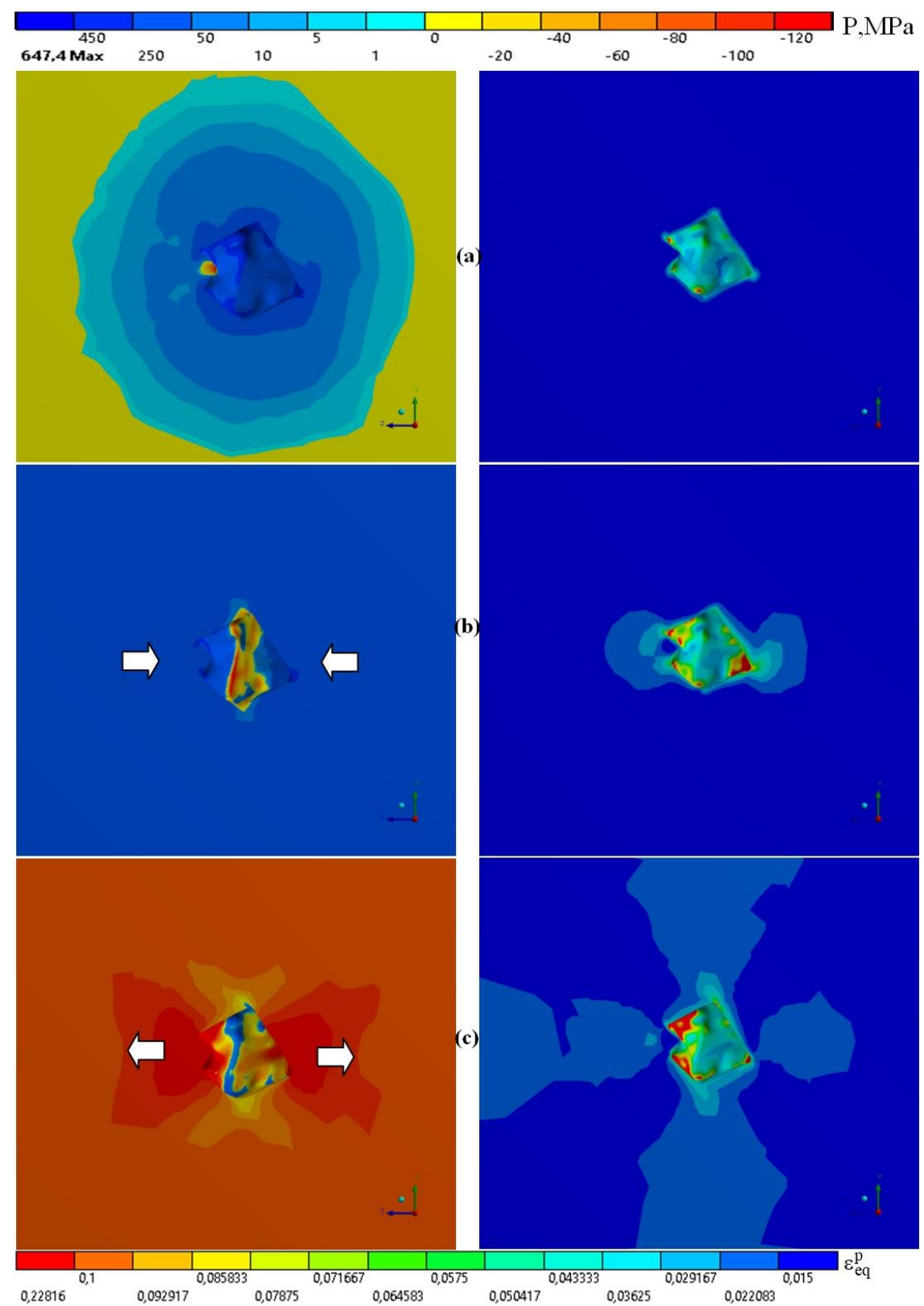

Fig. 4 Pressure and corresponding equivalent plastic strain patterns in the aluminum matrix under different conditions: cooling (a) and cooling followed by compression (b) or tension of the composite (c) 
For the case of cooling it is shown that the area of the particle influence on the stressed state in the matrix is on the order of an average particle diameter (Fig. 4a). Figure $6 \mathrm{a}$ is also a good illustration of the above conclusion that under the conditions of all-round compression a local bulk tension region (red color) is formed in the matrix only. It has to be noted that the equivalent stress in this region is minimal, and aluminum is not plastically deformed here (Fig. 4a).

In the cases of a combined thermomechanical loading, the external tensile or compressive loads would be added to already existing stresses in the material caused by cooling, thus forming the patterns differing from each other (Cf. Figs. 3e and $3 \mathrm{f}$ ). The main difference consists in the fact that the stresses in the C-type region are considerably higher in the tensile case than under compression. There is a more unexpected and exciting conclusion resulting from a comparison of the states formed during compression and tension for the cases where residual stresses are and are not taken into account. It is clear that the patterns in the C-type region under compression are very similar (Cf. Figs. $3 \mathrm{~b}$ and $3 \mathrm{e}$ ), while under tension they are quite different (Cf. Figs. 3d and 3f). Therefore, a preliminary qualitative conclusion is as follows: residual stresses have little effect on the stress concentration in the C-type region during compression and strongly affect it under tension. This conclusion is quite natural for the tensile case, though least important. It is natural because the compressive stresses, generated in the C-type region during cooling (Fig. 3c), are enhanced by the compressive loads appearing in this region during tension (Fig. 3d). It is least important, because these stresses are compressive, so the particle would not be fractured despite the high level of the equivalent stress in these regions. Hence, the first part of this interim conclusion becomes a preliminary general conclusion - residual stresses have little effect on the mechanical properties of the composites. At a first glance, this conclusion is odd. Seemingly, the compressive loads, developing in the particle during cooling (Fig. $3 \mathrm{c}$ ), would counteract the tensile stresses generated in the Ctype region in the course of subsequent uniaxial compression (Fig. $3 b$ ) and reduce the hazardous stress concentration in these areas. Then there is a question: why does not the concentration finally decrease (Cf. Figs. $3 \mathrm{~b}$ and $3 \mathrm{e}$ )? An answer to this question required a more detailed analysis of the evolution of the maximum stress concentration formed in the C-type region of the particle. The results of this analysis are aggregated in Fig. 5. The evolution of stress concentration during tension without preliminary cooling (without RSs) or during cooling followed by tension (with RSs) is depicted in red curves; compression or cooling followed by compression are shown by black curves. Zero strain corresponds to the state of the melt before cooling. There is all-round compression to about $-1.6 \%$, resulting from cooling, where the red and black curves coincide. Then from the state at $-1.6 \%$ the structure is additionally loaded by compression or tension. For the sake of convenience, the dependences for tension or compression without RSs also start at $-1.6 \%$. Additionally the stress concentration evolution in the matrix material near the C-type region is presented by circles for the cases of cooling followed by tension or compression of the composite.

It is clear that the stresses under tension are on the whole higher than under compression (compare the black and red curves in Fig. 5); note that the stress values in the entire range of composite elongations are by $1.5-4$ factors higher for the case where RSs are taken into account than where they are not (compare red triangles and squares in Fig. 5). For the above-described reasons, the plots under tension are less appealing from the practical perspective, since region $\mathrm{C}$ is under the conditions of bulk compression - 
pressure is positive in the entire range of strains (Fig. 5a, red curves). From the fundamental viewpoint, the following facts are interesting. In the initial stages of tensile loading after cooling, the stresses increase, then drop, and after that they start gradually increasing again. The sharp drop of the stresses results from plastic flow in the aluminum matrix, during which the stressed state is rearranged from all-round compression to uniaxial tension. As soon as this adjustment to the uniaxial tensile conditions is completed, the local compressive stresses in the $\mathrm{C}$-type region of the particle start monotonically increasing again. It is seen in Fig. 5 that the stress decreases in the aluminum matrix near the C-type region, which was caused by the rearrangement, then on further tension its decrease terminates, the stress remains constant, with its value being considerably lower than the yield point (Fig. 5b, red circles). This suggests that the matrix material in the C-type region after adaptation to the uniaxial tension conditions remains in the elastic stage of the deformation; it is in the 'silent' zone not suffering from any additional loads.

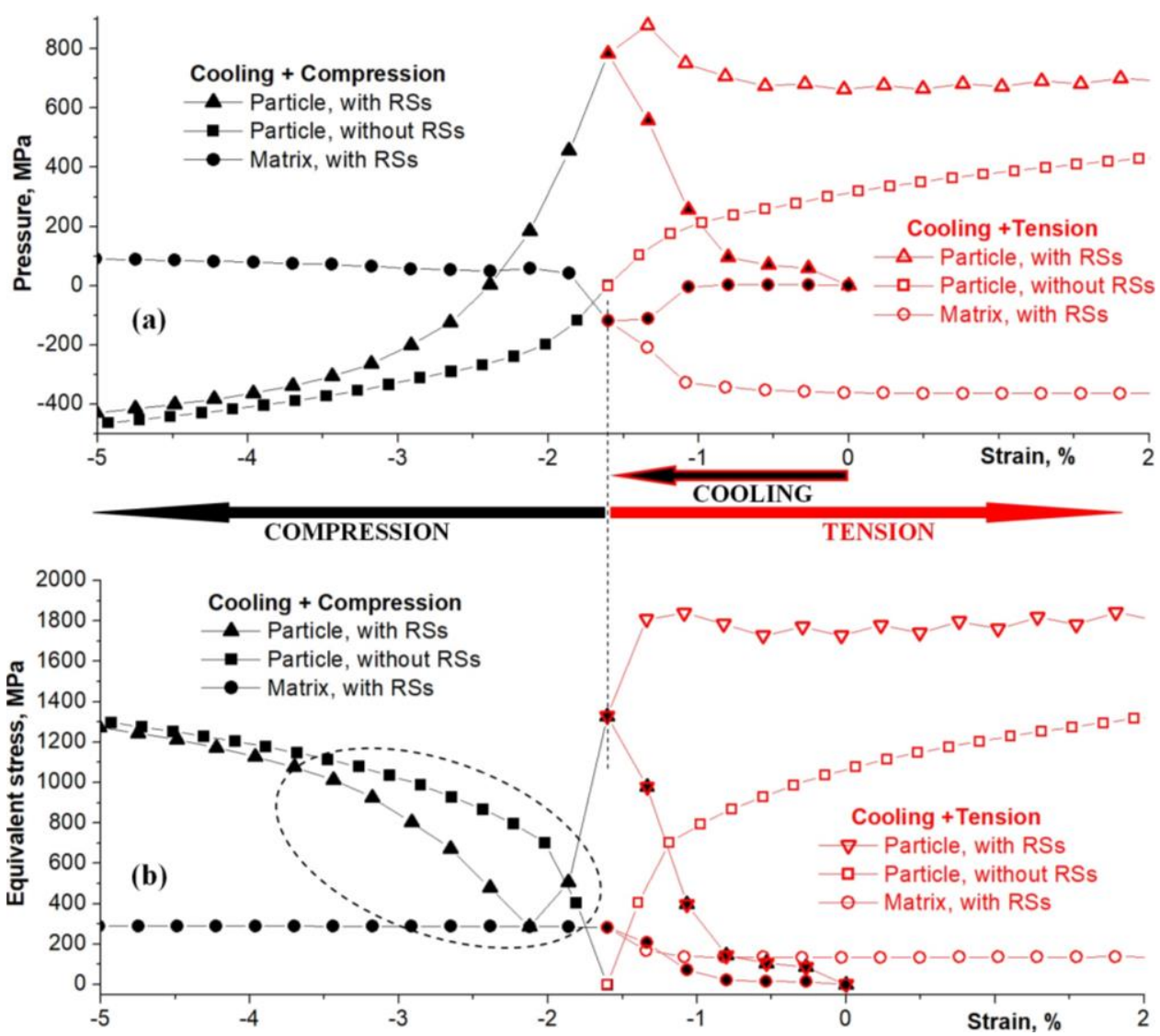

Fig. 5 Maximum pressure (a) and equivalent stress (b) in the C-type region of the particle shown in Fig. 3 and in the corresponding near-interface region of the matrix 
During cooling followed by external uniaxial compression, there is a completely different situation. During cooling the local compressive pressure increases in the C-type region (Fig. 5a, black-red triangles). Then after additional loading by uniaxial compression, the conditions of bulk tension start forming in this region. The pressure that increased during cooling begins sharply decreasing, while remaining to be positive, i.e. compressive (Fig. 5a, black triangles). At a certain point of compression equal to $-2.1 \%$, the pressure becomes negative and region $\mathrm{C}$ transfers into a state of bulk tension. Starting from this time point the equivalent stress stops decreasing and starts increasing (Fig. 5b, black triangles). This point corresponds to the minimum equivalent stress, about $250 \mathrm{MPa}$, which is by a factor of three lower than that under the conditions of compression without including residual stresses into account (Cf. black triangles and squares in Fig. 5b). Comparing the black curves with and without RSs being taken into consideration (Cf. black triangles and squares in Fig. 5 a), it is evident that the rate of pressure decrease in the initial stages of structure compression after cooling (Particle, with RSs in Fig. 5a) is considerably higher than if the structure was compressed from the initial state without preliminary cooling (Particle, without RSs in Fig. 5a). Upon further deformation, the rates of pressure drop equalize and these curves converge, demonstrating an equal level of stress concentration in the C-type local regions of bulk tension. As this goes on the aluminum matrix material undergoes plastic flow, maintaining the equivalent stress at a level of the current yield stress (Fig. 5b, black circles).

The residual stresses therefore play a positive role in the initial composite loading stages (up to 3-4\% compression), essentially reducing the level of hazardous stresses in the local regions of tension (indicated by a dashed circle in Fig. 5b) and do not strongly affect the stressed state at larger strain degrees.

\section{CONCLUSIONS}

An example of using the microstructure-based numerical simulation technique for investigating the formation and evolution of residual stresses in metal-ceramic composites has been discussed. A numerical model has been proposed for constructing three-dimensional structures of materials with particles of irregular shapes. Using the proposed technique, structures of composites and coatings of different scales have been designed. The details of strain localization and stress concentration in the interface regions have been investigated numerically during cooling from the melt to room temperature and subsequent uniaxial loading of the aluminum - titanium carbide composite.

The principal conclusions drawn from the numerical simulations are the following:

1. In the composites subjected to compression local regions of bulk tension are formed near the interfaces. In the cases of uniaxial compression such regions are observed both in the matrix and in the particles, while under all-round compression - in the matrix only. The volume fraction of tensile regions in the particles is larger and the concentration of stresses in them is lower during plastic flow in the matrix than in the case where the matrix deforms elastically. To sum up, fracture in metal-matrix composites occurs later and can be characterized by multiple cracking of the particles, while in ceramic composites it takes place earlier and is associated with propagation of isolated cracks. Stress concentration is by a factor of 1.5 times higher under all-round than under uniaxial compression. 
2. Under all-round compression up to $-1.6 \%$, which results from cooling of the composite from the melt to room temperature, local plastic strains are as large as $12 \%$.

3. The cooling-induced residual stresses play a positive role during subsequent mechanical loading of the composite to low strain degrees and only slightly influence its strength at the strains larger than $4 \%$.

Acknowledgement: The work was supported by the Russian Science Foundation (Project No. 1819-00273).

\section{REFERENCES}

1. Dimaki, A.V., Shilko, E.V., Dudkin, I.V., Psakhie, S.G., Popov, V.L., 2020, Role of adhesion stress in controlling transition between plastic, grinding and breakaway regimes of adhesive wear, Scientific Reports, 10, article number 1585 .

2. Aghababaei, R., Warner, D.H., Molinari, J.-F., 2017, Critical length scale controls adhesive wear mechanisms, Nature Communications, 7, 11816.

3. Popov, V., 2020, Coefficients of restitution in normal adhesive impact between smooth and rough elastic bodies, Reports in Mechanical Engineering, 1(1), pp. 103-109.

4. Li, Q., Voll, L., Starcevic, J., Popov, V.L., 2018, Heterogeneity of material structure determines the stationary surface topography and friction, Scientific Reports, 9, 14168

5. Gopinath Muvvala, Debapriya Patra Karmakar, Ashish Kumar Nath, 2017, Online assessment of TiC decomposition in laser cladding of metal matrix composite coating, Materials and Design, 121, pp. 310-320.

6. Yang-Feng, Tao, Jun, Li, Ying-Hao, Lv, Lie-Feng, Hu, 2017, Effect of heat treatment on residual stress and wear behaviors of the TiNi/Ti2Ni based laser cladding composite coatings, Optics and Laser Technology, 97, pp. 379-389.

7. Kadolkar, P.B., Watkins, T.R., De Hosson, J.Th.M., Kooi, B.J., Dahotre, N.B., 2007, State of residual stress in laser-deposited ceramic composite coatings on aluminum alloys, Acta Materialia, 55 (4), pp. 1203-1214.

8. Sravan Kumar Josyula, Suresh Kumar Reddy Narala, 2018, Study of TiC particle distribution in Al-MMCs using finite element modeling, International Journal of Mechanical Sciences, 141, pp. 341-358.

9. Ding Wang, Pratheek Shanthraj, Hauke Springer, Dierk Raabe, 2018, Particle-induced damage in Fe-TiB2 high stiffness metal matrix composite steels, Materials and Design, 160, pp. 557-571.

10. Leclerc, W., Haddad, H., Guessasma, M., 2018, On a discrete element method to simulate thermal-induced damage in 2D composite materials, Computers and Structures, 196, pp. 277-291.

11. Modesar Shakoor, Marc Bernacki, Pierre-Olivier Bouchard, 2018, Ductile fracture of a metal matrix composite studied using 3D numerical modeling of void nucleation and coalescence, Engineering Fracture Mechanics, 189, pp. 110-132.

12. Dariusz M. Jarząbek, Cezary Dziekoński, Wojciech Dera, Justyna Chrzanowska, Tomasz Wojciechowski, 2018, Influence of $\mathrm{Cu}$ coating of $\mathrm{SiC}$ particles on mechanical properties of $\mathrm{Ni} / \mathrm{SiC}$ co-electrodeposited composites, Ceramics International, 44, pp. 21750-21758.

13. Liang Ma, Cheng Huang, Kevin Dolman, Xinhu Tang, Jianjun Yang, Zheng Shi, Zhong-Sheng Liu, 2017, A method to calculate the bulk hardness of metal matrix composite using Hadfield steel reinforced with niobium carbide particles as an example, Mechanics of Materials, 112, pp. 154-162.

14. Moon Shik Park, Young W. Kwon, 2013, Elastoplastic micromechanics model for multiscale analysis of metal matrix composite structures, Computers and Structures, 123, pp. 28-38.

15. Shilko Evgeny V., Psakhie Sergey G.; Schmauder Siegfried, Popov Valentin L, Astafurov Sergey V., Alexey Smolin, 2015, Overcoming the limitations of distinct element method for multiscale modeling of materials with multimodal internal structure, Computational materials science, 102, pp. 267-285.

16. Chun Zhang, Zhiyong Cai, Yougen Tang, Richu Wang, Chaoqun Peng, Yan Feng, 2018, Microstructure and thermal behavior of diamond/Cu composites: Effects of surface modification, Diamond \& Related Materials, 86, pp. 98-108.

17. Zhang, J.F., Zhang, X.X., Wang, Q.Z., Xiao, B.L., Ma, Z.Y, 2018, Simulation of anisotropic load transfer and stress distribution in SiCp/Al /Al composites subjected to tensile loading, Mechanics of Materials, 122, pp. 96-103.

18. Sharma, N.K., Mishra, R.K., Sharma, S., 2016, 3D micromechanical analysis of thermo-mechanical behavior of Al2O3/Al metal matrix composites, Computational Materials Science, 115, pp. 192-201. 
19. Balokhonov, R.R., Romanova, V.A., Schmauder, S., Emelianova, E.S., 2019, A numerical study of plastic strain localization and fracture across multiple spatial scales in materials with metal-matrix composite coatings, Theoretical and Applied Fracture Mechanics, 101, pp. 342-355.

20. Balokhonov, R., Romanova , V., \& Zemlianov, A., 2021, A mesoscopic analysis of a localized shear band propagation effect on the deformation and fracture of coated materials, Reports in Mechanical Engineering, 2(1), pp. 6-22.

21. Balokhonov, R.R., Evtushenko, E.P., Romanova, V.A., Schwab, E.A., Bakeev, R.A., Emelyanova, E.S., Zinovyeva, O.S., Zinovyev, A.V., Sergeev, M.V., 2020, Formation of bulk tensile regions in metal matrix composites and coatings under uniaxial and multiaxial compression, Physical Mesomechanics, 23(2), pp. 135-146.

22. Balokhonov, R., Romanova, V., Kulkov, A., 2020, Microstructure-based analysis of deformation and fracture in metal-matrix composite materials, Engineering Failure Analysis, 110, 104412. 